\title{
Stability of non-time-reversible phonobreathers
}

\author{
J Cuevas $^{1}$, JFR Archilla ${ }^{2}$ and FR Romero ${ }^{3}$ \\ 1 Grupo de Física No Lineal. Departamento de Física Aplicada I, Escuela Politécnica Superior. \\ Universidad de Sevilla. Virgen de África 7, 41011 Sevilla, Spain \\ 2 Grupo de Física No Lineal. Departamento de Física Aplicada I, Escuela Técnica Superior de Ingeniería \\ Informática. Universidad de Sevilla. Avda. Reina Mercedes, s/n, 41012 Sevilla, Spain \\ 3 Grupo de Física No Lineal. Área de Física Teórica, Facultad de Física. Universidad de Sevilla. Avda. \\ Reina Mercedes, s/n, 41012 Sevilla, Spain \\ E-mail: jcuevas@us.es
}

\begin{abstract}
.
Non-time reversible phonobreathers are non-linear waves that can transport energy in coupled oscillator chains by means of a phase-torsion mechanism. In this paper, the stability properties of these structures have been considered. It has been performed an analytical study for low-coupling solutions based upon the so called multibreather stability theorem previously developed by some of the authors [Physica D 180 235]. A numerical analysis confirms the analytical predictions and gives a detailed picture of the existence and stability properties for arbitrary frequency and coupling.
\end{abstract}

PACS numbers: $63.20 . \mathrm{Ry}$

Submitted to: J. Phys. A: Math. Gen. 


\section{Introduction}

One of the subjects where a great deal of attention has been focused in the last two decades is the dynamics of nonlinear lattices. Intrinsic localized modes or discrete breathers is one of the most outstanding structures that arise in those lattices [1,2]. Discrete breathers are periodic and localized solutions whose existence is allowed by the interplay between discreteness and nonlinearity. The existence of those structures in networks of anharmonic oscillators (also known as Klein-Gordon lattices) was firstly proven at 1994 by MacKay and Aubry [3]. They established that discrete breathers can be continued from the anti-continuous limit (i.e. the limit where there is no coupling between the oscillators) to finite coupling as long as no integer multiple of the breather frequency resonates with the linear modes in the phonon band.

This theorem also demonstrates the existence of breathers with more than one excited sites, dubbed as multibreathers. These multibreathers receive special names in some cases; for instance, breathers with all their sites excited receive the name of phonobreathers. If all but one of the sites are excited the multibreather is called a dark breather [4], in analogy to the dark solitons existing in the Discrete Nonlinear Schrödinger (DNLS) equation $[5,6]$.

In order to observe experimentally discrete breathers, they have to be stable. The stability of one-site breathers (i.e. breathers with only one excited site) was firstly proven in $[7,8]$. It was not until 2003 where some of the authors of the present paper developed a method for determining the stability or instability of many kinds of multibreathers $[9,10]$ based on the Aubry's band theory developed in Ref. [7]. An alternative approach, introduced in [11], is based in previous work by MacKay et al [12,13,14]. Recently, the equivalence between both approaches has been proven [15].

Most of theoretical work related to discrete breathers has been carried on time-reversible solutions of Hamiltonian lattices. Non-time-reversible solutions have mostly been considered in dissipative lattices [16, $17,18,19]$, which have many experimental applications in chains of coupled pendula [20], micromechanical arrays [21], transmission lines [22, 23] and Josephson junction arrays [24, 25].

However, there have been very few approaches to non-time-reversible breathers or multibreathers in Hamiltonian lattices. The existence of one of such solutions was firstly proven in the pioneer work of MacKay and Aubry [3]. It was restricted to phonobreathers where there is a constant phase difference between adjacent sites. These solutions can transport energy by means of phase torsion and can be viewed as nonlinear phonons or phasons. Later on, Aubry [7] demonstrated the existence of a generic non-timereversible breather, independent on the excited sites. The proof was also extended to inhomogeneous lattices (e.g. with vacancies) and vortices. It was also proven that phonobreathers with phase torsion generate a stationary flux, and that time-reversible phonobreathers cannot transport energy. Finally, Cretegny and Aubry numerically demonstrate $[26,27]$ the existence of non-time-reversible phonobreathers in homogenous and inhomogeneous (with vacancies) 1-D lattices and vortices and breather "rivers" (i.e. percolating clusters of breathers connecting two boundaries submitted to phase torsion) in $2 \mathrm{D}$ square lattices. They also sketch some properties of the flux and its stability in 1D lattices. Further applications of this theory can be found in many publications on discrete vortices in DNLS [28, 29, 30, 31, 32] and Klein-Gordon [15, 33] lattices.

The aim of this paper is twofold. On the one hand, we will prove by making use of the techniques developed in $[9,10]$, a result mentioned in passing on Ref. [26]: the stability of phonobreathers depends on the phase difference between adjacent sites and the phonobreather frequency. On the other hand, we will make an analysis of the stability and flux dependence on the system parameters for two kind of potentials in Klein-Gordon lattices, checking the validity of the analytical predictions.

The paper is organized as follows: in Section 2 we introduce the model equations; section 3 deals with the analytical results regarding the stability for low coupling; in order to check these results, we make an exhaustive numerical analysis of the existence and stability of phonobreathers at finite coupling in Section 4; finally, we present our conclusions and some possible extension of this work in Section 5 . 


\section{Model setup}

\subsection{Dynamical equations and energy flux}

We a consider a Klein-Gordon chain of oscillators with nearest-neighbours harmonic coupling. The dynamical equations are of the form:

$$
\ddot{u}_{n}+V^{\prime}\left(u_{n}\right)+\varepsilon\left(2 u_{n}-u_{n+1}-u_{-1}\right)=0 \quad n=1, \ldots, N
$$

where the variables $u_{n}$ are the displacements with respect to the equilibrium positions, $V\left(u_{n}\right)$ is the on-site potential, $N$ is the number of oscillators, and $\varepsilon>0$ is the coupling constant.

We consider two paradigmatic cases of on-site potentials: Morse (soft) potential

$$
V(u)=\frac{1}{2}[\exp (-u)-1]^{2}
$$

and $\phi^{4}$ (quartic) hard potential

$$
V(u)=\frac{1}{2} u^{2}+\frac{1}{4} u^{4}
$$

We look for non-time-reversible solutions of the dynamical equations (1) with all the particles excited, so that the difference between the phases of the temporal oscillations of two nearest-neighbours lattice sites is a constant $\alpha$. Thus, there is a phase torsion between the boundaries of the lattice of value $\tau=N \alpha$. In order to fit the periodic boundary conditions, $\tau=2 \pi m$ with $m \in \mathbb{Z}$, and, in consequence, $\alpha=2 \pi m / N$. Hence, we are dealing with an anharmonic plane wave (or a phonobreather) with a wave number equal to $\alpha$ that transmits an energy flux along the chain of oscillators [26].

Let us remark the similarities of non-time-reversible phonobreathers with q-breathers, which are localized solutions in the reciprocal space (see [34] for FPU and [35] for DNLS lattices). q-breathers can be generally considered as the superposition of a finite number of normal modes. The solutions considered in the present paper, i.e. phonobreathers, are also localized in the reciprocal space, so they can be cast as a special case of q-breathers, consisting only of a normal mode characterized by the wavenumber $\alpha$.

In order to calculate phonobreather solutions we make use of methods based on the anti-continuous limit $[3,36]$, that is, an orbit of frequency $\omega_{\mathrm{b}}$ for each isolated oscillator is calculated and the coupling constant is subsequently varied with a path-following (Newton-Raphson) method. In this paper, we have used a Fourier space implementation of the dynamical equations.

Fourier space methods are based on the fact that the solutions are $T_{\mathrm{b}}$-periodic (For a detailed explanation of these methods, the reader is referred to Refs. [37, 38, 39]). Thus, they can be expressed in terms of a truncated Fourier series expansion:

$$
u_{n}(t)=\sum_{k=-k_{m}}^{k_{m}} z_{k, n} \exp \left(i k\left(\omega_{\mathrm{b}} t+\alpha\right)\right)
$$

with $k_{m}$ being the maximum of the absolute value of the running index $k$. In the numerics, $k_{m}$ has been chosen as 13. After the introduction of (4), the dynamical equations (1) transform into a set of $N \times\left(2 k_{m}+1\right)$ algebraic equations where the variables are $Z \equiv\left\{z_{k, n}\right\}$ :

$$
F_{k, n} \equiv-\omega_{\mathrm{b}}^{2} k^{2} z_{k, n}+\mathcal{F}_{k}\left[V^{\prime}\left(u_{n}\right)\right]+\varepsilon\left(2 z_{k, n}-z_{k, n-1}-z_{k, n+1}\right)=0
$$

Here, $\mathcal{F}_{k}$ denotes the Discrete Fourier Transform:

$$
\mathcal{F}_{k}\left[V^{\prime}\left(u_{n}\right)\right]=\frac{1}{2 k_{m}+1} \sum_{j=-k_{m}}^{k_{m}} V^{\prime}\left(u\left(t_{j}\right)\right) \exp \left(-i k \omega_{\mathrm{b}} t_{j}\right),
$$



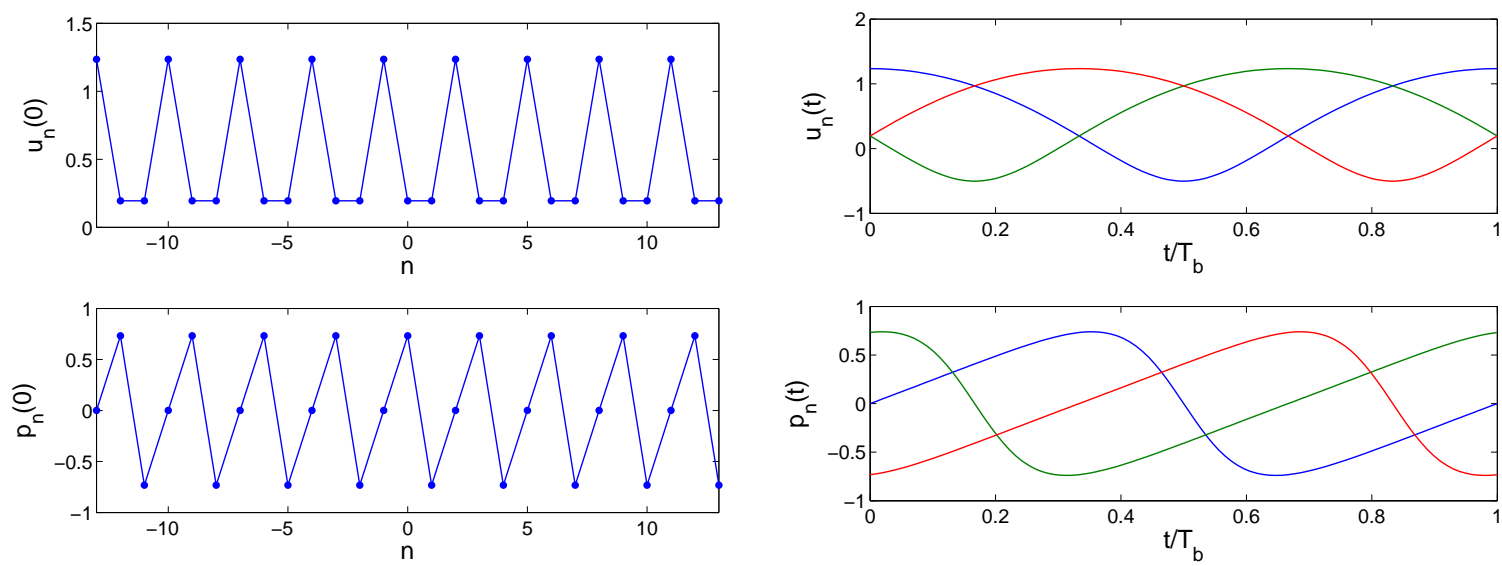

Figure 1. Position $u_{n}(t)$ and linear momentum $p_{n}(t)=\dot{u}_{n}(t)$ profiles (left panel) and time evolution (right panel) for a phonobreather with $\omega_{\mathrm{b}}=0.8, \varepsilon=0.05, \alpha=2 \pi / 3$ and a Morse potential. Different curves in right panels stand for different lattice sites. Notice that there are only 3 curves in those panels because this is the spatial periodicity of the phonobreather.

where $t_{j}$ is a sample of times that must be chosen equally spaced:

$$
t_{j}=\frac{2 \pi j}{\omega_{\mathrm{b}}\left(2 k_{m}+1\right)}, \quad j=-k_{m}, \ldots,+k_{m},
$$

and $u_{n}\left(t_{j}\right)$ is calculated from the Fourier coefficients $z_{k, n}$ by means of the Inverse Discrete Fourier Transform:

$$
u_{n}\left(t_{j}\right)=\sum_{k=-k_{m}}^{k_{m}} z_{k, n} \exp \left(i k \omega_{\mathrm{b}} t_{j}\right) .
$$

Fourier space methods provide with an analytical form of the Jacobian $\mathcal{J} \equiv \partial F / \partial Z$, whose elements are $\left\{\partial F_{k, n} / \partial z_{k^{\prime}, n^{\prime}}\right\}$. Figs. 1 and 2 show the profiles and time evolution of two examples of phonobreathers $\ddagger$ with different phases and potentials.

Phonobreathers can transport energy by means of the phase torsion mechanism, and consequently, there is a stationary energy flux as long as $\alpha \neq 0, \pi[7]$. With the aid of the Fourier series expansion, the energy flux can be expressed as:

$$
J_{n \rightarrow m}=-J_{m \rightarrow n}=\frac{\epsilon}{T_{\mathrm{b}}} \int_{0}^{T_{\mathrm{b}}} u_{n}(t) \dot{u}_{m}(t) \mathrm{d} t=-i \omega_{\mathrm{b}} \epsilon \sum_{k} k z_{k, n} z_{-k, m}
$$

Phonobreathers are characterized by a constant energy density at each lattice site. Thus, it must be fulfilled that $z_{k, n+1}=z_{k, n} \exp (i k \alpha)$ with $z_{k, n}=z_{-k, n}^{*}$ and the flux between neighbouring sites is homogeneous and fulfills:

$$
J_{0} \equiv J_{n, n+1}=2 \omega_{\mathrm{b}} \varepsilon \sum_{k \geq 1} k\left|z_{k, n}\right|^{2} \sin (k \alpha) \quad \forall n
$$

$\ddagger$ Notice that hereby we will omit, for simplicity, the non-time reversible character of the solutions 

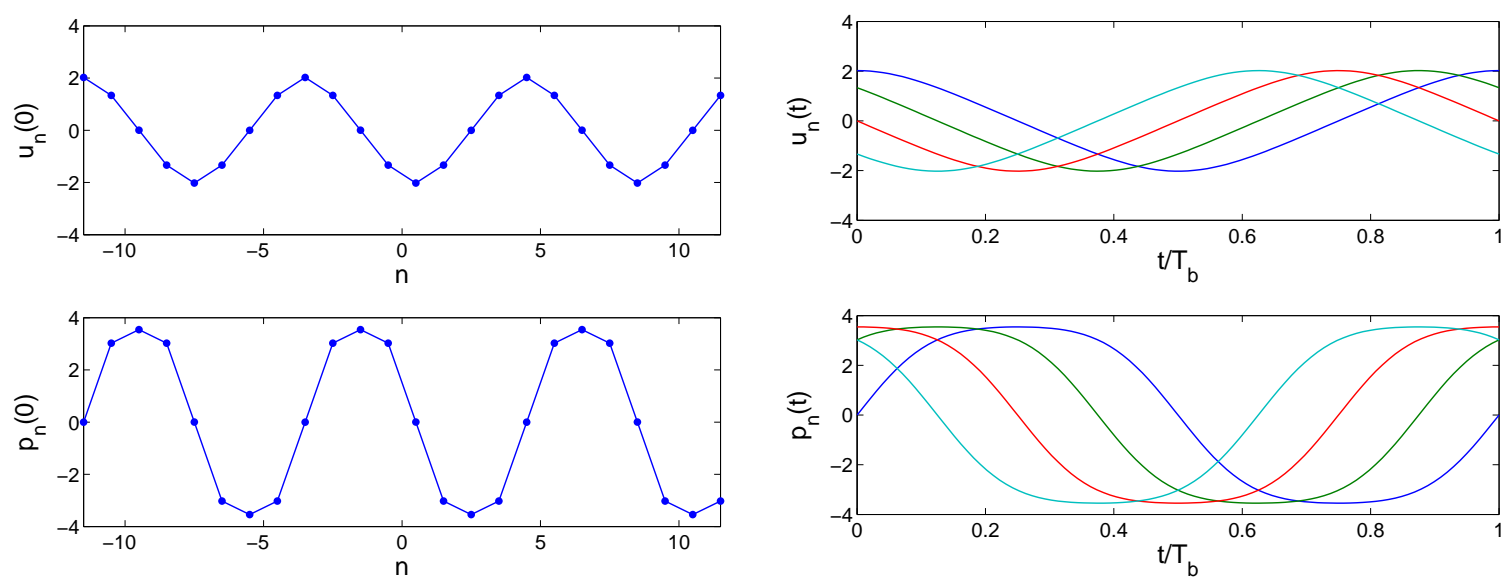

Figure 2. Position $u_{n}(t)$ and linear momentum $p_{n}(t)=\dot{u}_{n}(t)$ profiles (left panels) and time evolution (right panel) for a phonobreather with $\omega_{\mathrm{b}}=2, \varepsilon=0.05, \alpha=\pi / 4$ and a $\phi^{4}$ potential. Different curves in right panels stand for different lattice sites. Notice that there are only 4 curves in those panels because this is the spatial periodicity of the phonobreather.

\subsection{Linear stability equations}

In order to study the linear stability of phonobreathers, we introduce a small perturbation $\xi_{n}$ to a given solution $u_{n 0}$ of Eq. (1) according to $u_{n}=u_{n 0}+\xi_{n}$. Then, the equations satisfied to first order on $\xi_{n}$ is:

$$
\ddot{\xi}_{n}+V^{\prime \prime}\left(u_{n 0}\right) \xi_{n}+\varepsilon\left(2 \xi_{n}-\xi_{n+1}-x_{n-1}\right)=0,
$$

or, in a more compact form:

$$
\mathcal{N}(\{u(t)\}) \xi=0
$$

where $\mathcal{N}(\{u(t)\})$ is known as the Newton operator. In order to study the orbital stability analysis of the relevant solution, a Floquet analysis can be performed if there exists $T_{b} \in \mathbb{R}$ so that the map $u_{n}(0) \rightarrow u_{n}\left(T_{\mathrm{b}}\right)$ has a fixed point [7]. Then, the stability properties are given by the spectrum of the Floquet operator $\mathcal{M}_{0}$ (whose matrix representation is the monodromy) defined as:

$$
\left(\begin{array}{c}
\left\{\xi_{n}\left(T_{\mathrm{b}}\right)\right\} \\
\left\{\dot{\xi}_{n}\left(T_{\mathrm{b}}\right)\right\}
\end{array}\right)=\mathcal{M}_{0}\left(\begin{array}{c}
\left\{\xi_{n}(0)\right\} \\
\left\{\dot{\xi}_{n}(0)\right\}
\end{array}\right)
$$

The $2 N \times 2 N$ monodromy eigenvalues $\Lambda=\exp (\mathrm{i} \theta)$ are dubbed the Floquet multipliers. This operator is symplectic and real, which implies that there is always a pair of multipliers (corresponding to the phase and growth modes) at 1 and that the eigenvalues come in duplets $\{\Lambda, 1 / \Lambda\}$ if they are real or quadruplets $\left\{\Lambda, 1 / \Lambda, \Lambda^{*}, 1 / \Lambda^{*}\right\}$ if they are complex. Consequently, if the phonobreather is stable, all the eigenvalues lie on the unit circle.

Equation (12) can be seen as the eigenequation of the Newton operator for the eigenvalue $E=0$. Then, the eigenequations for the Newton operator are:

$$
\mathcal{N}(\{u(t)\}) \xi=E \xi \rightarrow \ddot{\xi}_{n}+V^{\prime \prime}\left(u_{n 0}\right) \xi_{n}+\varepsilon\left(2 \xi_{n}-\xi_{n+1}-x_{n-1}\right)=E \xi_{n}
$$

The Newton operator is periodic in time, and, consequently, its eigenvectors fulfill the Floquet-Bloch theorem. This theorem implies that the $E$-eigenvalues spread bringing about a set of bands $\mathcal{E}_{\nu}=\mathcal{E}_{\nu}(\theta)$, where $\theta$ can be chosen in the First Brioullin zone, i.e. $\theta \in[-\pi, \pi]$. The set of eigenvalues $E_{\nu}(\theta)$ with $\theta$ real 
is denoted as the $\nu$-th band. The bands are associated to stable solutions as long as $\theta_{\nu} \in \mathbb{R}$. The values of $\theta(E)$ can be obtained by diagonalizing the matrix $\mathcal{M}_{E}$, which is obtained in a similar fashion to Eq. (13) but integrating Eq. (14) for each value of $E$. The monodromy corresponds obviously to $E=0$ and, consequently, the Floquet arguments correspond to $\theta_{\nu}(0)$. Thus, a solution is stable if there are be $2 N$ bands that either cross the $E=0$ axis or are tangent to it. More details on this theory, called Aubry's band theory can be found in Ref. [7].

\section{Analytical results}

In this section, we will show some analytical predictions about the stability of phonobreathers at low coupling. To this end, we start by recalling previous results established by some of the authors for multibreathers with an arbitrary number of excited sites and continue by applying these results to the prediction of the stability properties of phonobreathers with Morse and $\phi^{4}$ potentials at low coupling.

\subsection{Previous results}

For the sake of completeness, we recall in this subsection some previous results on the Multibreathers Stability Theorem (MST) proposed in Ref. [9]. For more details, the reader is also referred to Refs. [10, 15].

The MST refers to Klein-Gordon systems of the form (1) and estimates the displacement experienced by Aubry's bands when the coupling parameter $\varepsilon$ is switched on.

Suppose that $u_{n}^{0}$ is a $T_{\mathrm{b}}$-periodic solution at the anti-continuous limit $(\varepsilon=0)$, with $p$ excited oscillators and $N-p$ ones at rest $\left(u_{n}^{0}=0\right)$. At this limit, there are $p$ degenerated bands tangent to the $E=0$ axis at $(E, \theta)=(0,0)$. Their curvature is positive (negative) for soft (hard) on-site potentials. The MST can predict the displacement of the minimum of this bands $\{\Delta E\}_{i=0}^{p-1}=\{E\}_{i=0}^{p-1}=\varepsilon\{\lambda\}_{i=0}^{p-1}$, with $\{\lambda\}$ being the set of eigenvalues for the perturbation matrix $Q$. The (non-zero) non-diagonal elements of the $p \times p$ perturbation matrix $Q$ in reduced form (see below) are defined as

$$
Q_{n, n \pm 1}=-\frac{1}{\mu_{n} \mu_{n \pm 1}} \int_{-T_{\mathrm{b}} / 2}^{T_{\mathrm{b}} / 2} \dot{u}_{n}^{0} \dot{u}_{n \pm 1}^{0} \mathrm{~d} t
$$

with $\mu_{n}=\sqrt{\int_{-T / 2}^{T / 2}\left(\dot{u}_{n}^{0}\right)^{2} \mathrm{~d} t}$. Only the indexes corresponding to the excited oscillators are considered and they are renumbered them from 1 to $p$. If we considered all the oscillators, the matrix $Q$ would be in full form. Each oscillator at rest adds a row and a column of zeros and, therefore, a zero eigenvalue, which is not relevant for the stability properties.

The diagonal elements are given by

$$
Q_{n, n}=-\frac{\mu_{n+1} Q_{n, n+1}+\mu_{n-1} Q_{n, n-1}}{\mu_{n}} .
$$

With these definitions, we reproduce here the multibreather stability theorem:

Generalized MST Given a Klein-Gordon system, Eq. (1), a specific multibreather solution at zero coupling $\left\{u_{n}^{0}\right\}, u(t)$ the corresponding solution at low and positive coupling, $\{\lambda\}_{i=0}^{p-1}$ the eigenvalues of the reduced, perturbation matrix $Q$, with only one zero, then:

The solution $u(t)$ is stable if:

a) The on-site potentials are soft and there is not any positive value in $\left\{\lambda_{i}\right\}_{i=0}^{p-1}$.

b) The on-site potentials are hard and there is not any negative value in $\left\{\lambda_{i}\right\}_{i=0}^{p-1}$. 
We can summarize the stability properties in the following way. If $S=1$ means stability and $S=-1$ instability, $H=1$ corresponds to a hard on-site potential, and $H=-1$ to a soft one, and we define $\operatorname{sign}(Q)=1$ if all the eigenvalues of $Q$ but a zero one are positive and $\operatorname{sign}(Q)=-1$ if they are negative except for the zero one, then

$$
S=H \times \operatorname{sign}(Q) .
$$

It there are eigenvalues of different signs, the multibreather is always unstable. It is important to take into account that there is always a zero eigenvalue due to a global phase mode. If there is more than one zero eigenvalue, the stability theorem can only predict the instability in the case that there exists at least one eigenvalue $\lambda$ that leads to $S=-1$ in the previous equation (changing $\operatorname{sign}(Q)$ for $\operatorname{sign}(\lambda)$ ), but not the stability, as the 0 -eigenvalue is degenerate.

\subsection{Application to phonobreathers}

We apply hereby the theory recalled in the previous subsection for the system given by Eq. (1). Consequently, we must construct the reduced perturbation matrix $Q$ whose elements are given by Eqs. (15) and (16). The functions $u_{n}^{0}(t)$ that appear in those equations are the solutions of the isolated oscillators submitted to the potentials $V\left(u_{n}\right)$, i.e. the solutions of the equations:

$$
\ddot{u}_{n}^{0}+V^{\prime}\left(u_{n}^{0}\right)=0,
$$

Let $I_{n m}$ be defined as:

$$
I_{n, m}=\int_{-T_{\mathrm{b}} / 2}^{T_{\mathrm{b}} / 2} \dot{u}_{n}^{0}(t) \dot{u}_{m}^{0}(t) \mathrm{d} t
$$

and the parameters $\vartheta_{n, m}$ as:

$$
\vartheta_{n, m}=\frac{I_{n, m}}{\sqrt{I_{n, n} I_{m, m}}} .
$$

Substituting Eq. (4) into (19)-(20) and taking into account that the phase difference between neighbouring sites $\alpha$ is constant, we get that

$$
\vartheta_{n, n \pm 1} \equiv \vartheta=\frac{\sum_{k} k^{2} z_{k}^{2} \cos (k \alpha)}{\sum_{k} k^{2} z_{k}^{2}} .
$$

With the aid of the $\vartheta$ parameter, the $Q$ matrix can be written as

$$
Q=\vartheta Q^{(R)}
$$
lattice:

where $Q^{(R)}$ is the $Q$ matrix for a time-reversible phonobreather (i.e. with $\alpha=0$ ) in an homogeneous

$$
Q_{n, m}^{(R)}=2 \delta_{n, m}-\left(\delta_{m, n+1}+\delta_{n, n-1}\right), \quad n=1, \ldots N
$$

Thus, the eigenvalues of our problem are given by $\lambda=\vartheta \lambda_{R}$ with $\lambda_{R}$ the eigenvalues of the time-reversible lattice. These eigenvalues are given by $\lambda_{R, n}=4 \sin ^{2}(n \pi / N)$ with $n=0, \ldots, N-1$ [9]; consequently:

$$
\lambda_{n}=4 \vartheta \sin ^{2} \frac{n \pi}{N} \quad n=0, \ldots, N-1 .
$$

Thus, $\operatorname{sign}\left(\lambda_{n}\right)=\operatorname{sign}(\vartheta)$. Following Eq. (17), the phonobreather is stable for a soft potential if $\vartheta<0$ and, for a hard potential, if $\vartheta>0$. In the case of a symmetric on-site potential, $z_{2, \nu}=0, \forall \nu \in \mathbb{Z}$, and consequently, $\vartheta(\pi / 2)=0$.

We calculate below the expression of $\vartheta$ for a Morse and a hard $\phi^{4}$ potential: 

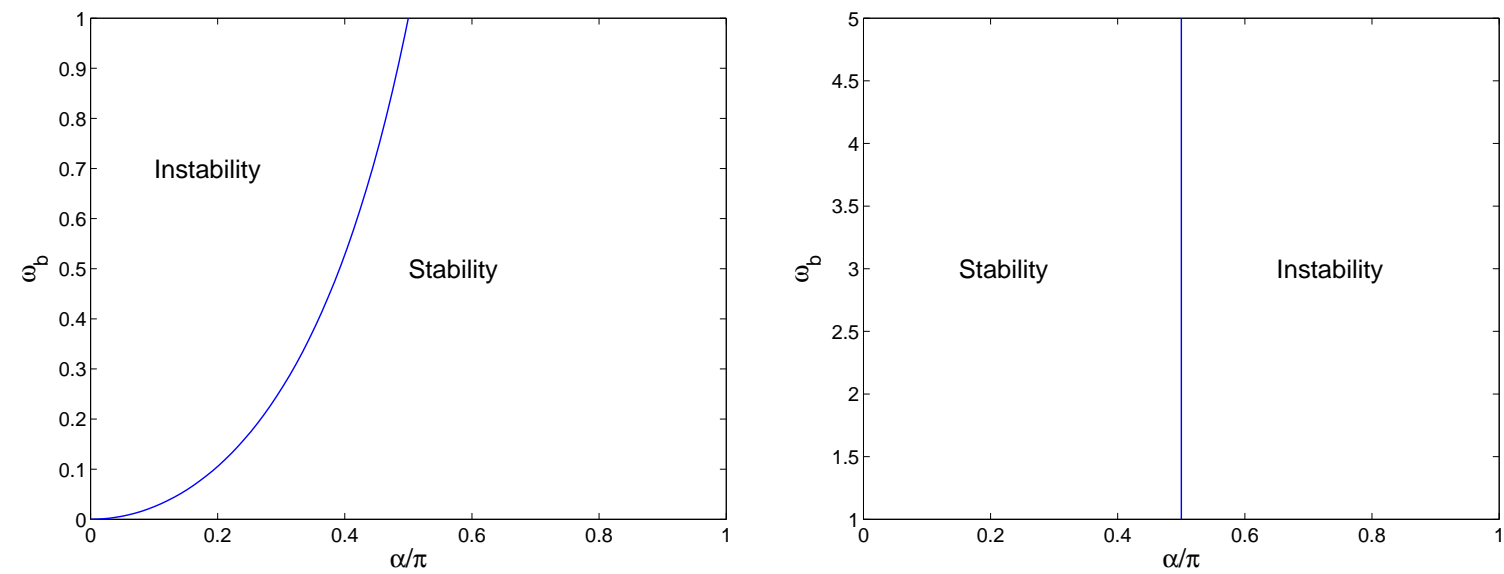

Figure 3. Stability and instability regions predicted by the MST for Morse (left) and $\phi^{4}$ (right) potentials. Recall that, for the Morse $\left(\phi^{4}\right)$ potential, instability implies $\vartheta<0(\vartheta>0)$.

3.2.1. Morse potential The orbits of Eq. (18) are given by [9]:

$$
u(t)=\log \frac{1-\sqrt{1-\omega_{\mathrm{b}}^{2}} \cos \omega_{\mathrm{b}} t}{\omega_{\mathrm{b}}^{2}},
$$

The Fourier coefficients are:

$$
z_{0, n}=\log \frac{1+\omega_{\mathrm{b}}}{2 \omega_{\mathrm{b}}^{2}} \quad ; \quad z_{k, n}=-\frac{(-1)^{k+1}}{k}\left(\frac{1-\omega_{\mathrm{b}}}{1+\omega_{\mathrm{b}}}\right)^{k / 2},
$$

Thus, we can write:

$$
\vartheta=\frac{\sum_{k=1}^{\infty} r^{k} \cos (k \alpha)}{\sum_{k=1}^{\infty} r^{k}}
$$

with

$$
r=\frac{1-\omega_{\mathrm{b}}}{1+\omega_{\mathrm{b}}} .
$$

Using [40], the sums can be performed, leading to:

$$
\vartheta=\frac{(1-r)(\cos \alpha-r)}{1-2 r \cos \alpha+r^{2}} .
$$

Thus,

$$
\operatorname{sign}(\vartheta)=\operatorname{sign}(\cos \alpha-r),
$$

In consequence, $\vartheta<0$ (i.e. the phonobreather is stable) if

$$
\cos \alpha<r\left(=\frac{1-\omega_{\mathrm{b}}}{1+\omega_{\mathrm{b}}}\right) .
$$

The main consequence of this condition is that, for a given value of $\alpha$, there exists a critical value of $\omega_{\mathrm{b}}$ below which the phonobreather is unstable. This critical value is:

$$
\omega_{\mathrm{b}, \mathrm{c}}=\frac{1-\cos \alpha}{1+\cos \alpha}=\tan ^{2} \frac{\alpha}{2}
$$


Consequently, as $\omega_{\mathrm{b}}<1$ for oscillators with Morse potential, if $\alpha>\pi / 2$ phonobreathers are always stable, independently of the frequency.

Fig. 3(left) shows the predictions of the MST for the Morse potential as a function of $\omega_{\mathrm{b}}$ and $\alpha$. For $\omega_{\mathrm{b}}=\omega_{\mathrm{b}, \mathrm{c}}(\alpha), \vartheta=0$ and the degeneracy of $\lambda_{n}$ cannot be removed; consequently, the MST would not be able to predict the (in)stability in that case.

3.2.2. $\phi^{4}$ potential The orbit of an oscillator submitted to a $\phi^{4}$ hard potential is given by [41]:

$$
u_{n}(t)=\sqrt{\frac{2 \kappa^{2}}{1-2 \kappa^{2}}} \mathrm{cn}\left(\frac{\omega_{\mathrm{b}} t}{\sqrt{1-2 \kappa^{2}}}, \kappa\right)=\sqrt{\frac{2 \kappa^{2}}{1-2 \kappa^{2}}} \mathrm{cn}\left(\frac{2 K(\kappa)}{\pi} \omega_{\mathrm{b}} t, \kappa\right),
$$

where cn is a Jacobi elliptic function of modulus $\kappa_{n}$ and $K(\kappa)$ is the complete elliptic integral of the first kind defined as:

$$
K(\kappa)=\int_{0}^{\pi / 2} \frac{\mathrm{d} x}{\sqrt{1-\kappa^{2} \sin ^{2} x}} .
$$

The breather frequency $\omega_{\mathrm{b}}$ is related to the modulus $\kappa$ through:

$$
\omega_{\mathrm{b}}=\frac{\pi}{2 \sqrt{1-2 \kappa^{2}} K(\kappa)} .
$$

In order to calculate $\vartheta$, it is better to use Eq. (20). Taking into account that $\vartheta$ depends only on the phase difference, we can write:

$$
J_{n m}=\frac{4 \kappa^{2} K(\kappa) \omega_{\mathrm{b}}^{3}}{\pi\left(1-2 \kappa^{2}\right)} \int_{-2 K(\kappa)}^{2 K(\kappa)} \mathrm{d} x \operatorname{sn}(x) \operatorname{sn}(x+a) \operatorname{dn}(x) \operatorname{dn}(x+a),
$$

with $a=2 K(\kappa) \alpha / \pi$. This integral can be evaluated applying [42, identity 171]:

$$
\begin{aligned}
& \kappa^{2} \operatorname{sn}(x) \operatorname{dn}(x) \operatorname{sn}(x+a) \operatorname{dn}(x+a)= \\
& -\operatorname{cs}(a) \operatorname{ns}(a)\left(1+\operatorname{dn}^{2}(a)\right)+\operatorname{cs}(a) \operatorname{ns}(a)\left(\operatorname{dn}^{2}(x)+\operatorname{dn}^{2}(x+a)\right)- \\
& \operatorname{ds}(a)\left(\operatorname{cs}^{2}(a)+\operatorname{ns}^{2}(a)\right)[\mathrm{Z}(x+a)-\mathrm{Z}(x)-\mathrm{Z}(a)],
\end{aligned}
$$

where $\mathrm{Z}(x)$ is the Jacobian elliptic $\mathrm{Z}$ function [43]. Then, the value of $\vartheta$ is given by:

$$
\vartheta=3 \frac{\operatorname{cs}(a) \operatorname{ns}(a)\left[2 E(\kappa)-K(\kappa)\left(1+\mathrm{dn}^{2}(a)\right)\right]-\mathrm{ds}(a)\left(\operatorname{cs}^{2}(a)+\mathrm{ns}^{2}(a)\right) \mathrm{Z}(a)}{\left(-1+2 \kappa^{2}\right) E(\kappa)+\left(1-\kappa^{2}\right) K(\kappa)}
$$

where $E(\kappa)$ is the complete elliptic function of the second kind:

$$
E(\kappa)=\int_{0}^{\pi / 2} \sqrt{1-\kappa^{2} \sin ^{2} x} \mathrm{~d} x .
$$

Three relevant values of $\vartheta$ correspond to $\alpha=\pi, \alpha=\pi / 2, \alpha=0$ which lead to $\vartheta=1, \vartheta=0$ and $\vartheta=-1$, respectively. This result can be straightforwardly determined from Eq. (21). An analytic determination of the sign of $\vartheta$ for other values of $\alpha$ is not possible. However, it is possible to visualize numerically that the only zero of $\vartheta(\alpha)$ takes place at $\alpha=\pi / 2$ being $\vartheta<0(\vartheta>0)$ for $\alpha<\pi / 2(\alpha>\pi / 2)$. In consequence, phonobreathers with a $\phi^{4}$ hard potential are stable as long as $\alpha<\pi / 2$.

Fig. 3(right) summarizes the predictions of the MST for the $\phi^{4}$ potential as a function of $\omega_{\mathrm{b}}$ and $\alpha$. For $\alpha=\pi / 2, \vartheta=0$ and the degeneracy of $\lambda_{n}$ cannot be removed, and consequently, the MST could not predict (in)stability. 

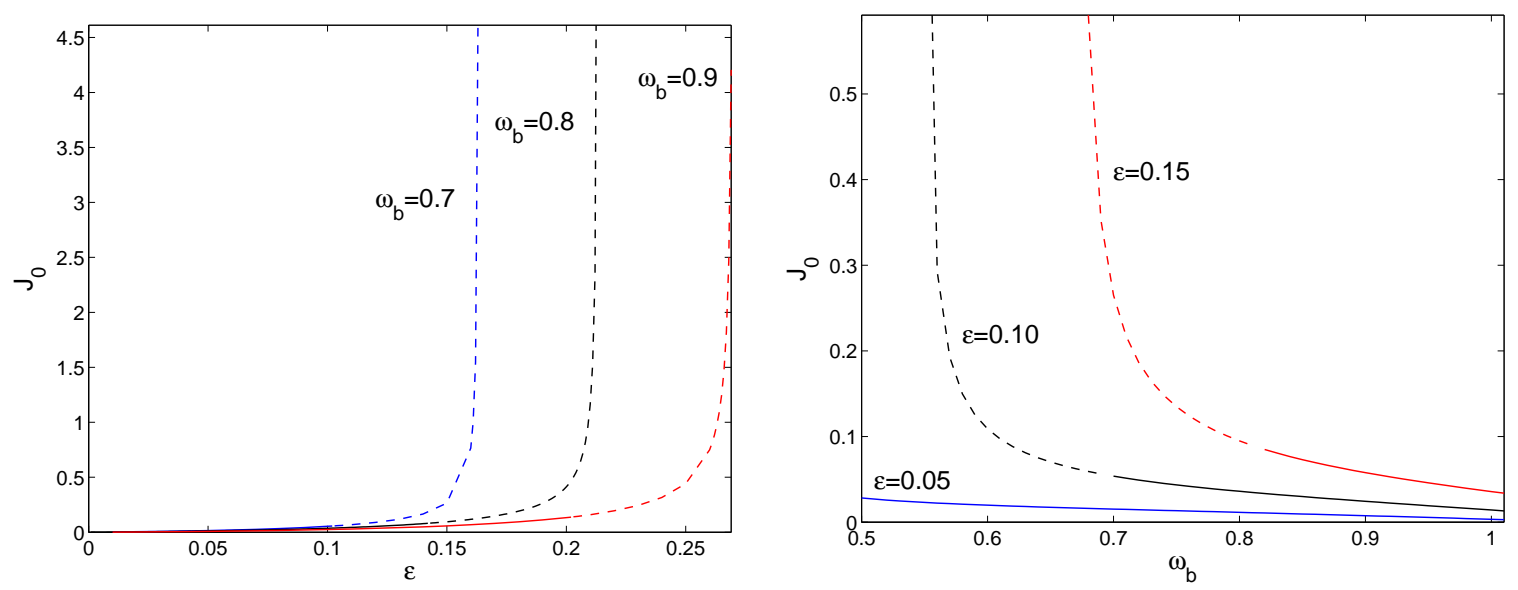

Figure 4. Flux between nearest-neighbours for phonobreathers with $\alpha=2 \pi / 3$ and a Morse potential. Dashed lines represent unstable solutions, for which the flux diverges above (below) a critical value of $\varepsilon$ $\left(\omega_{\mathrm{b}}\right)$.

\section{Numerical results}

The analytical results of the previous section concern to an infinitesimally small coupling. In this section, we explore the existence and stability properties for an arbitrary coupling constant and determine the range of validity of the predictions of the multibreather stability theorem.

\subsection{Morse potential}

Before undertaking the existence and stability analysis of the phonobreather, we show the properties of the energy flux. Fig. 4 depicts the dependence of $J_{0}$ with respect to $\epsilon$ and $\omega_{\mathrm{b}}$ for $\alpha=2 \pi / 3$; the qualitative behaviour displayed in the figure is generic for any value of $\alpha$. It is observed that for the Morse potential, the flux increases (decreases) with the coupling constant (frequency). There is a critical value of $\varepsilon\left(\omega_{\mathrm{b}}\right)$ above (below) which phonobreathers do not exist. For this critical value, the flux diverges. This fact is used in Appendix A to calculate the dependence of the critical value of $\varepsilon$ with $\omega_{\mathrm{b}}$.

Additionally, a full stability analysis has been performed by varying $\varepsilon$ and $\omega_{\mathrm{b}}$ for a Morse potential and choosing two different values of the phase difference $\alpha=2 \pi / 3$ and $\alpha=4 \pi / 9$. In the first case, phonobreathers are stable for small $\varepsilon$ and become unstable above a critical value of $\varepsilon$. In the second case, phonobreathers are unstable for small $\varepsilon$ as long as $\omega_{\mathrm{b}}>\omega_{\mathrm{b}, \mathrm{c}}$; the MST predicts that $\omega_{\mathrm{b}, \mathrm{c}}=0.7041$, which fits quite well with the numerics; in addition, for large enough $\varepsilon$, the phonobreather becomes stable, losing the stability again when $\varepsilon$ is increased further. As explained above, phonobreathers cease to exist above a critical value of $\varepsilon$ because the flux diverges. This critical value of $\varepsilon$ has been calculated in Eq. (A.6):

$$
\varepsilon_{c}=\frac{\omega_{\mathrm{b}}^{2}}{4 \sin ^{2}(\alpha / 2)}
$$

Fig. 5 depicts the existence and stability ranges for phonobreathers with the above mentioned parameters whereas Fig. 6 shows the dependence with respect to $\varepsilon$ of the arguments and moduli of Floquet multipliers for several examples. From these figures, it can be deduced that instabilities and stabilities arise by means of cascades of Neimark-Sacker bifurcations (also known as Krein crunches). An analysis of the dynamics shows that those instabilities lead to the disappearance of the phase torsion and the time and spatial periodicities of the phonobreathers. 

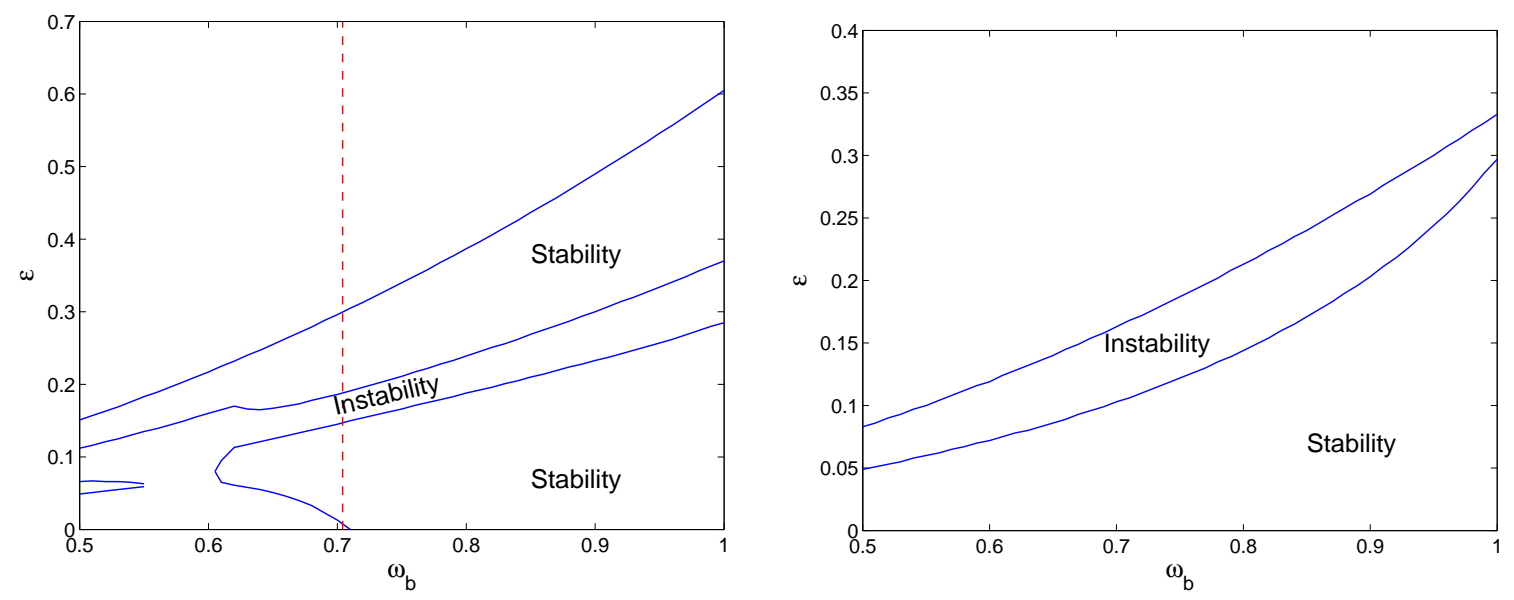

Figure 5. $\left(\varepsilon-\omega_{\mathrm{b}}\right)$ plane showing the existence and stability of phonobreathers with $\alpha=4 \pi / 9$ (left) and $\alpha=2 \pi / 3$ (right) in a Morse potential. Dashed line in left panel corresponds to the MST prediction (i.e. stable at the right of the line and unstable at the left) for low coupling.

We have checked the analytical predictions of MST regarding to Aubry's bands displacement. To this end, we show in Fig. 7 the bands for a phonobreather with small $\varepsilon$ whereas Fig. 8 shows the comparison of the predicted vertical displacement given by the MST with the numerical value. We can observe that, contrary to time-reversible multibreathers (where the displacement is purely vertical), there is a diagonal movement of the bands. In addition, there is a horizontal splitting of the bands that preserves the mirror symmetry of the bands with respect to $E$-axis. It is also found an excellent agreement with the analytical predictions even for high coupling when $\alpha=2 \pi / 3$ (the displacement is almost lineal), whereas for $\alpha=4 \pi / 9$ there is a serious discrepancy as the bands displacement is non-monotonic and non-linear.

\subsection{Hard $\phi^{4}$ potential}

The analysis of the previous case can also be done for the hard $\phi^{4}$ potential. We start by showing in Fig. 9 the properties of the flux for $\alpha=\pi / 4$ and $\alpha=3 \pi / 2$; the qualitative behaviour displayed in the figure is generic for any value of $\alpha$. Contrary to the Morse case, the flux has a non-monotonical behavior, increasing with $\varepsilon$ for small coupling and decreasing for large coupling; additionally, the flux is zero for zero coupling and for a critical value of $\varepsilon$. However, the flux increases with the frequency. As in the Morse case, this behaviour allowed us to calculate the critical coupling as a function of the frequency, as shown in Appendix A.

Let us show the results regarding to the stability. Analogously to the time-reversible breathers, there exist no stability exchange bifurcations and the stability for any arbitrary $\varepsilon$ is the same as for small coupling except for $\alpha=\pi / 2$. Thus, for $\alpha>\pi / 2(\alpha<\pi / 2)$, phonobreathers stability is the same as in the $\alpha=\pi$ $(\alpha=0)$ case. The only unknown appears at $\alpha=\pi / 2$, where the multibreathers stability theorem fails. In that case, numerical calculations demonstrate that the stability range depends both on $\varepsilon$ and $\omega_{\mathrm{b}}$. Fig. 10 displays the dependence of the Floquet multipliers with respect to $\varepsilon$ for $\alpha=2 \pi / 3, \alpha=\pi / 2$ and $\alpha=\pi / 4$. In all cases, for a given $\omega_{\mathrm{b}}$, there is a critical value of $\varepsilon$ where the continuation fails. At this value the flux vanishes, contrary to the Morse potential, for which the flux diverges. This critical value of $\varepsilon$ has been calculated in Eq. A.5:

$$
\varepsilon_{c}=\frac{\omega_{\mathrm{b}}^{2}-1}{4 \sin ^{2}(\alpha / 2)}
$$



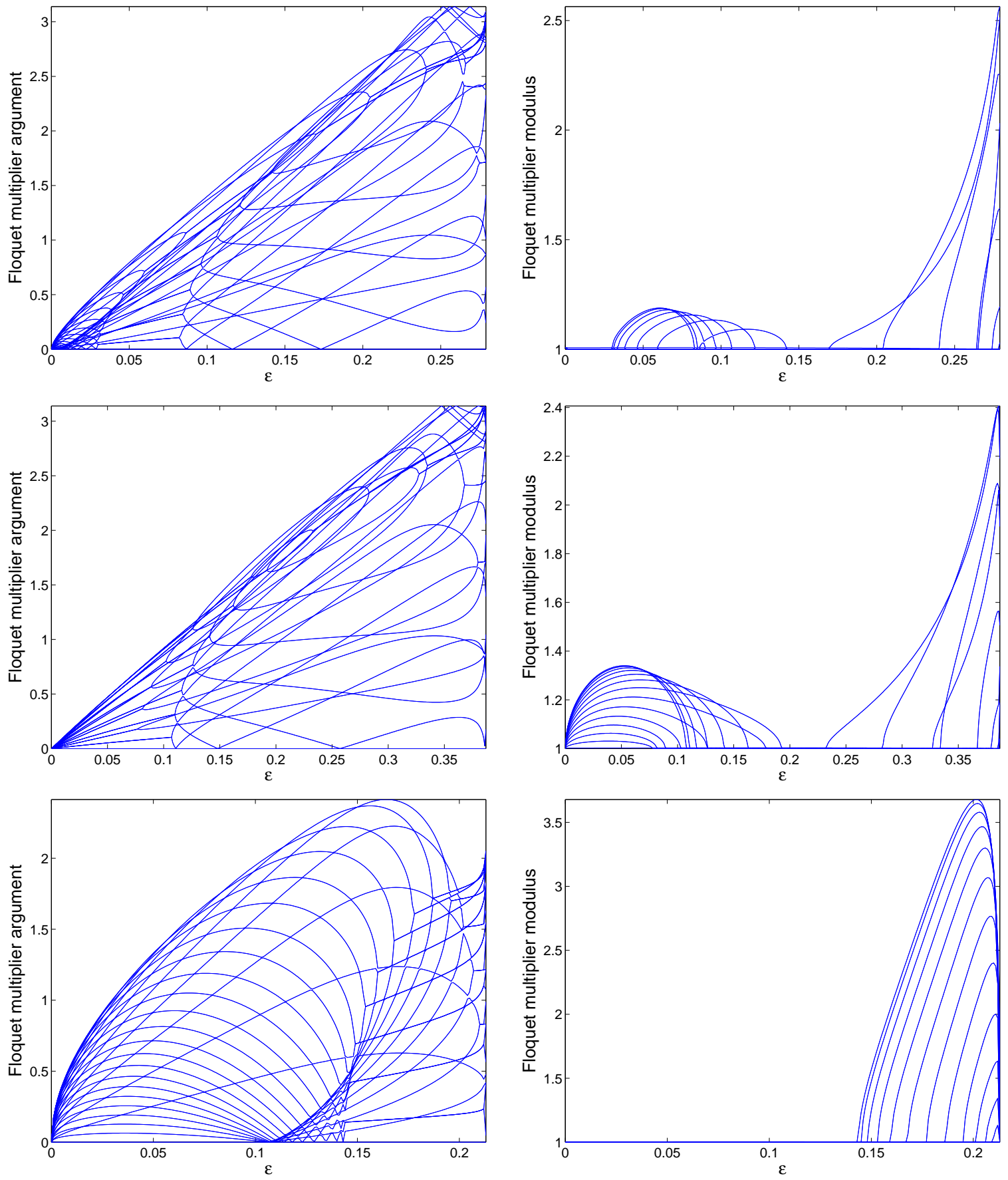

Figure 6. Dependence of the Floquet multipliers with respect to $\varepsilon$ for phonobreathers in a Morse potential. Parameters are: $\omega_{\mathrm{b}}=0.68$ and $\alpha=4 \pi / 9$ (top panels), $\omega_{\mathrm{b}}=0.8$ and $\alpha=4 \pi / 9$ (middle panels) and $\omega_{\mathrm{b}}=0.8$ and $\alpha=2 \pi / 3$ (bottom panels). 

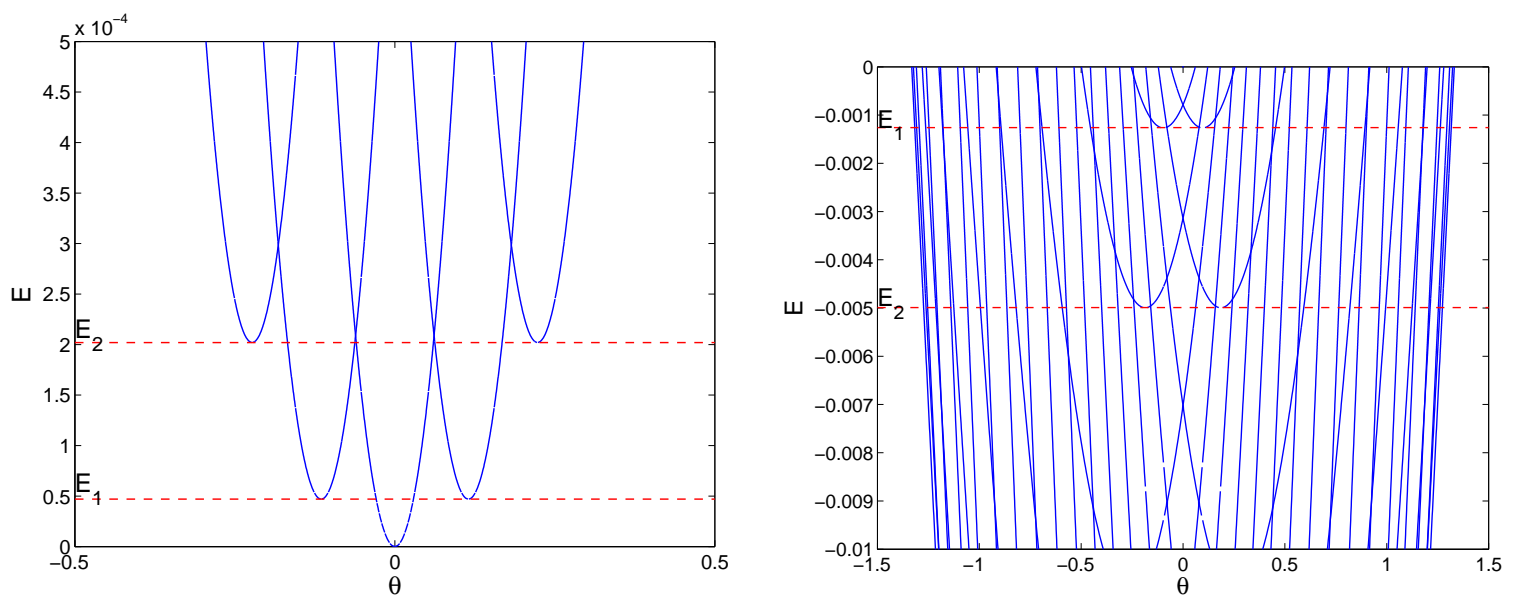

Figure 7. Bands for a phonobreather with $\varepsilon=0.05, \omega_{\mathrm{b}}=0.8$ and $\alpha=4 \pi / 9$ (left) and $\alpha=2 \pi / 3$ (right) and a Morse potential. The number of particles is $N=27$.
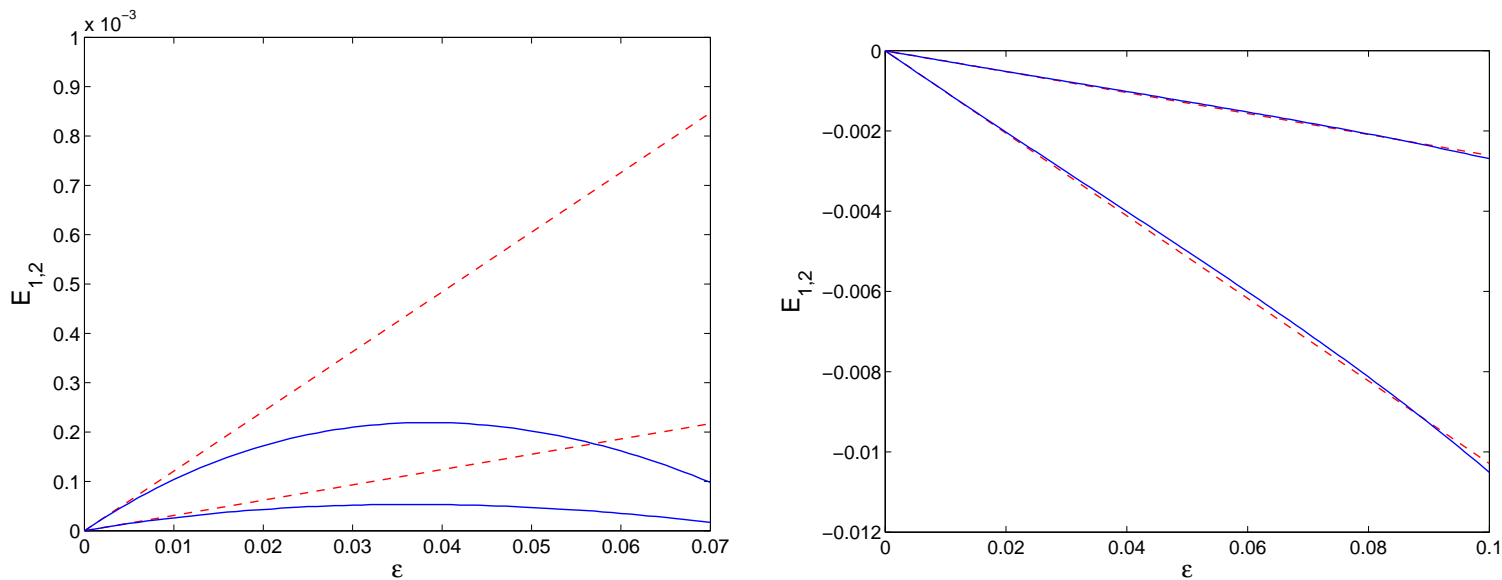

Figure 8. Dependence of the location of the minima of the two bands closest to $E=0$ (represented by $E_{1}$ and $E_{2}$ ) with respect to $\varepsilon$ for phonobreathers in a Morse potential with $\alpha=4 \pi / 9$ (left) and $\alpha=2 \pi / 3$ (right). In both cases, $\omega_{\mathrm{b}}=0.8$. Dashed lines correspond to the MST predictions and full lines to the numerical values. Solutions at left, where the discrepancy is high, are unstable for small but finite coupling.

Fig. 11 shows the existence range for those values of $\alpha$. It can be deduced that the existence range is enlarged when $\alpha$ decreases. Besides, for $\alpha=\pi / 2$ it is observed that the stability properties depends on the frequency and coupling and that phonobreathers are always unstable for $\omega_{\mathrm{b}} \leq 3$.

Fig. 12 and Fig. 13 show, respectively, examples of bands and the MST predictions, for $\alpha=2 \pi / 3$ and $\alpha=\pi / 4$. There is an excellent agreement of the numerical results with the analytical predictions. Additionally, Fig. 14 focuses on the $\alpha=\pi / 2$ case with $\omega_{\mathrm{b}}=2$. From the last figure, we can observe that the first and second minima of the bands adjust respectively to the parabolas $-0.015509 \varepsilon^{2}$ and $-0.056476 \varepsilon^{2}$. This fact confirms that a second order perturbation theory is needed in order to predict the stability properties for $\alpha=\pi / 2$. 

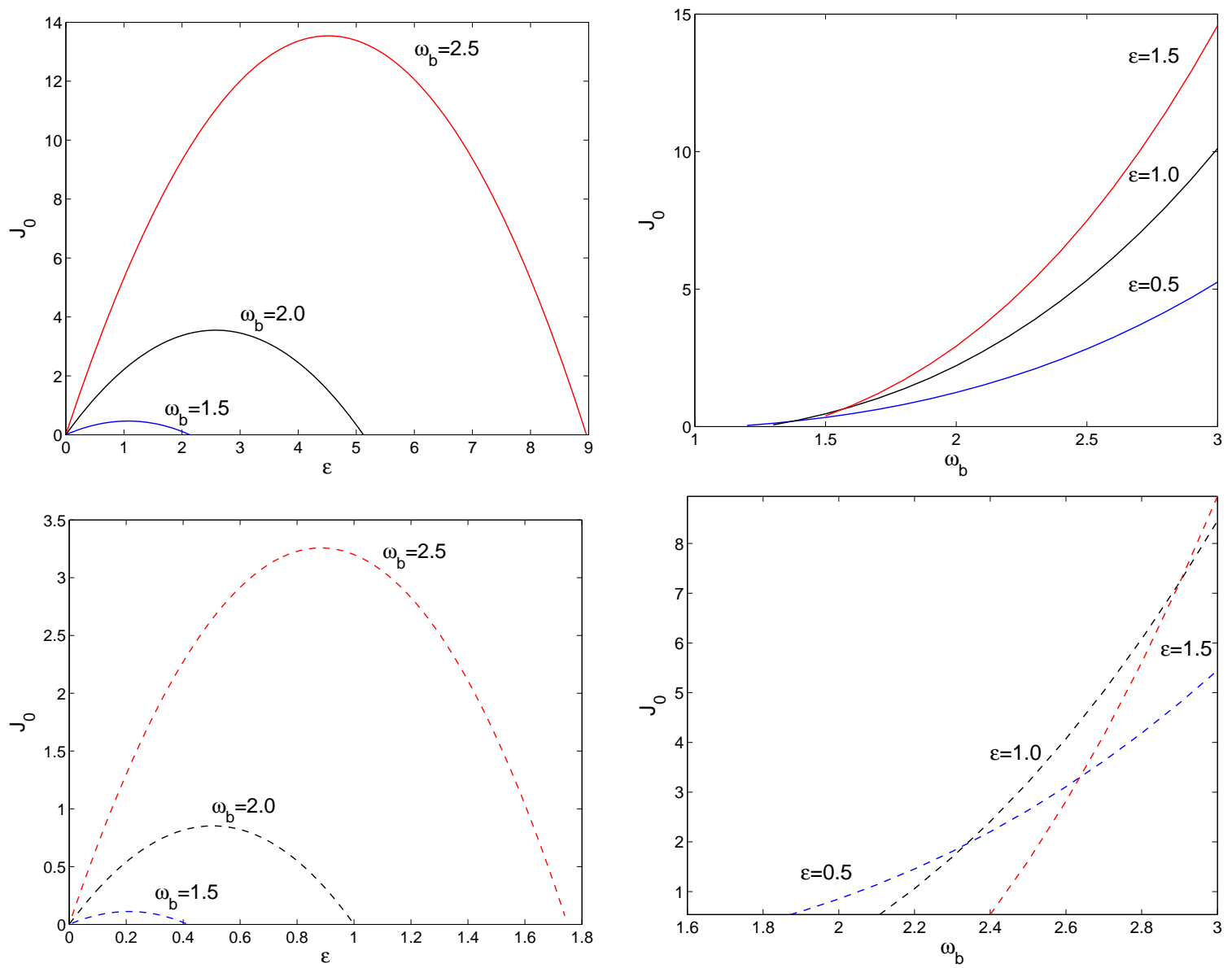

Figure 9. Flux between nearest-neighbours for phonobreathers in a $\phi^{4}$ potential with $\alpha=\pi / 4$ (top panels) and $\alpha=3 \pi / 2$ (bottom panels). Dashed lines represent to unstable solutions. Note that the flux tends to zero when the continuation in $\varepsilon$ fails.

\section{Conclusions}

The study carried out in this paper has been twofold: on the one hand, analytical predictions on the stability of non-time reversible phonobreathers has been established by making use of the multibreathers stability theorem; on the other hand, a numerical study on the existence and stability properties of those phonobreathers has been performed.

The multibreathers stability theorem predicts that there is a critical value of the phase difference in phonobreathers that separates stable and unstable states. This critical value depends on the phonobreather frequency for lattices with harmonic coupling and Morse on-site potential whereas, for the $\phi^{4}$ potential, the critical value is $\pi / 2$ and does not depend on the frequency. At this particular value of the phase, the theorem cannot make any prediction about the (in)stability.

The analytical findings have been confirmed by means of numerical analysis, showing in addition that the stability changes above a given value of the coupling constant. It has also been confirmed that, in most cases, the Aubry's band displacement predicted by the multibreather stability theorem for small coupling, is valid even for relatively high coupling. Apart from this, it has been observed that phonobreathers cease 

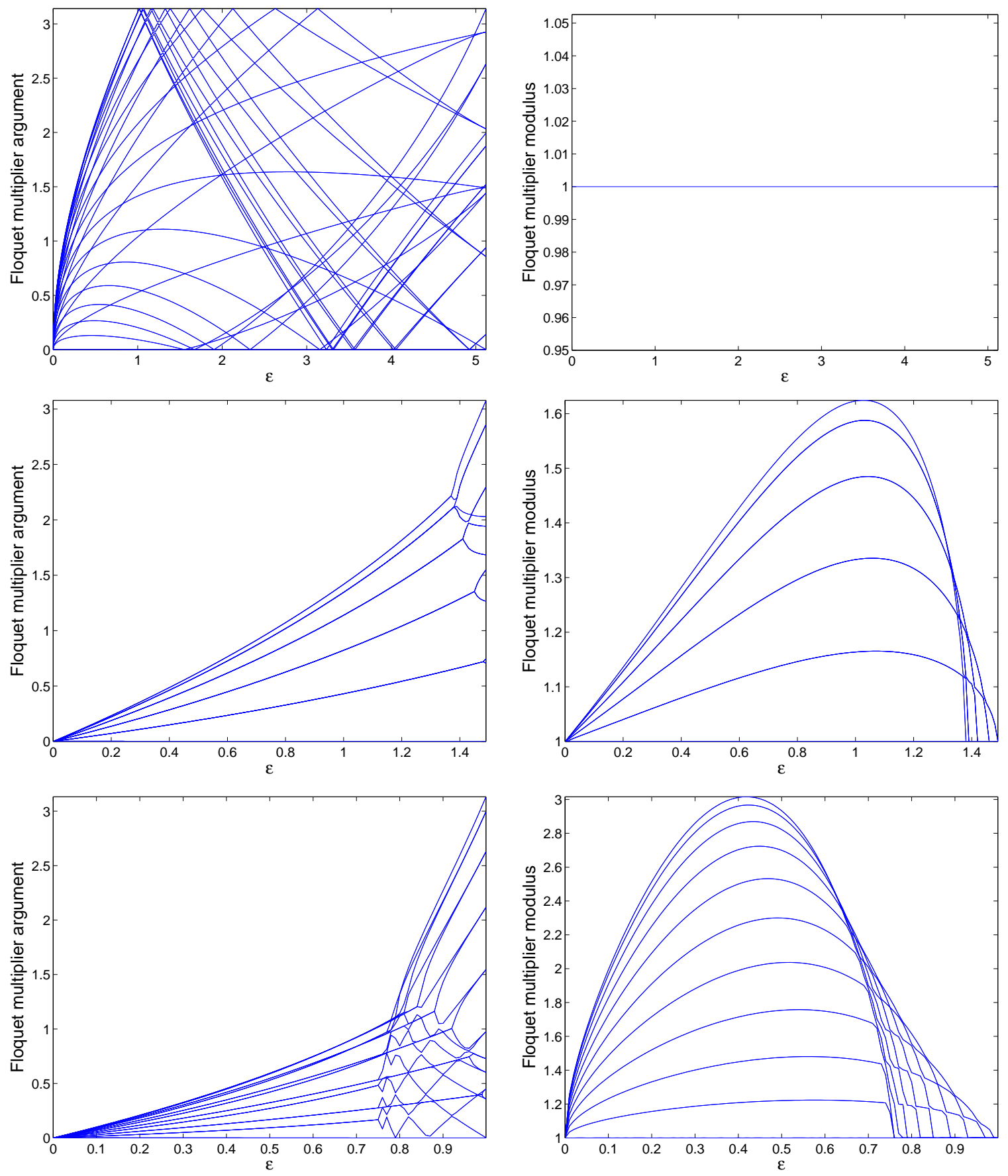

Figure 10. Dependence of the Floquet multipliers with respect to $\varepsilon$ for phonobreathers in a $\phi^{4}$ potential. Parameters are: $\omega_{\mathrm{b}}=2$ and $\alpha=2 \pi / 3$ (top panels), $\omega_{\mathrm{b}}=2$ and $\alpha=\pi / 2$ (middle panels) and $\omega_{\mathrm{b}}=2$ and $\alpha=\pi / 4$ (bottom panels). 

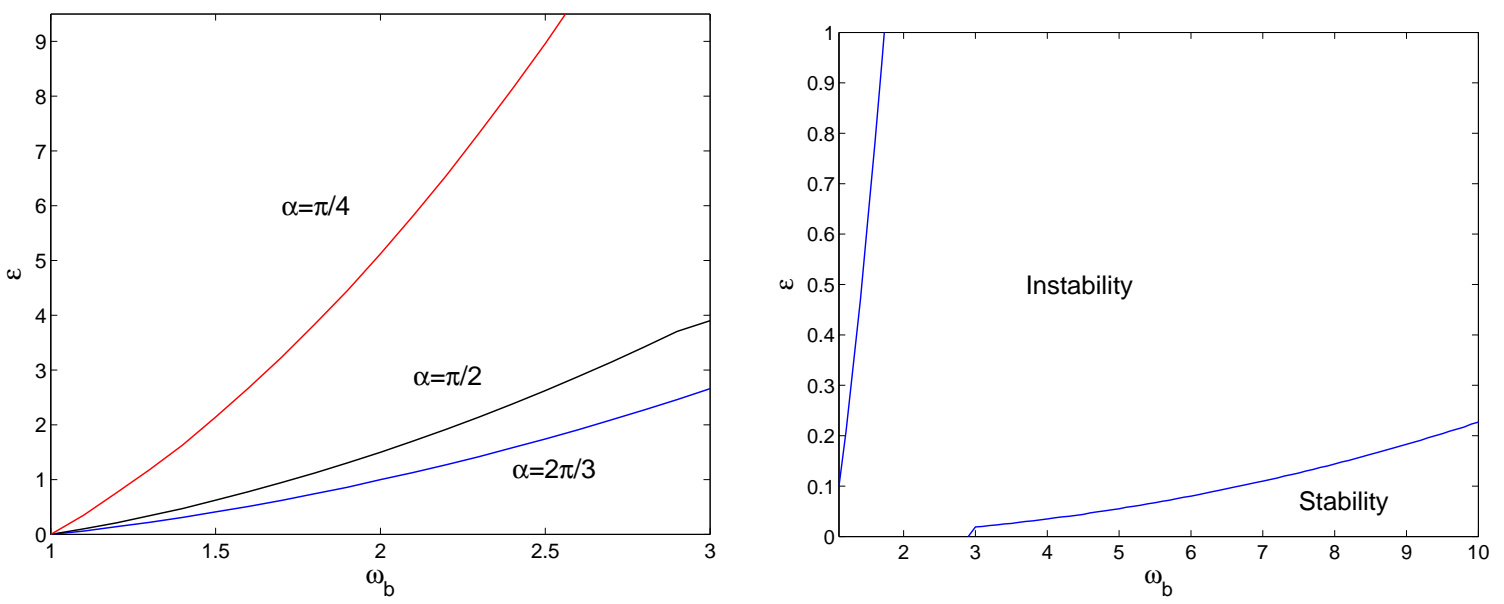

Figure 11. (Left) $\varepsilon-\omega_{\mathrm{b}}$ plane showing the existence of phonobreathers $\phi^{4}$ potential. (Right) shows the existence and stability range for phonobreathers with $\alpha=\pi / 2$.
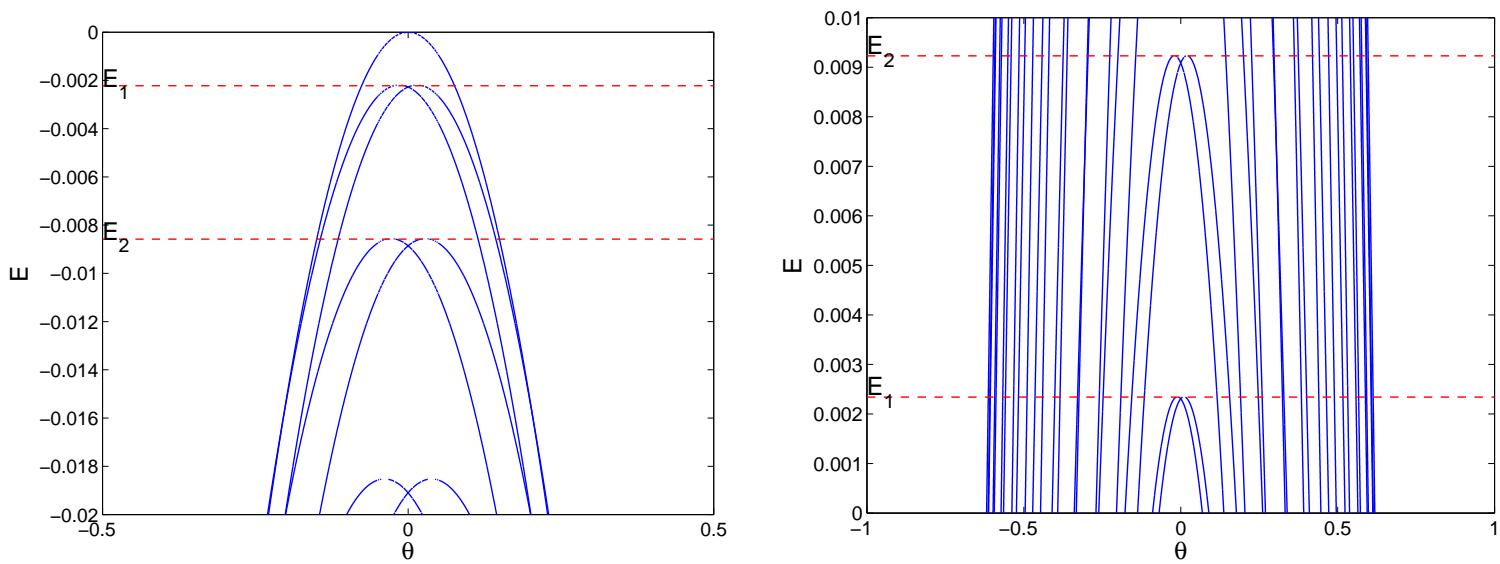

Figure 12. Bands for a phonobreather with $\varepsilon=0.05, \omega_{\mathrm{b}}=2$ and $\alpha=2 \pi / 3$ (left) and $\alpha=\pi / 4$ (right) and a $\phi^{4}$ potential. The number of particles is $N=21$ (left) and $N=24$ (right).

to exist above a critical value of the coupling. This value coincides with a flux divergence for the Morse potential or a flux vanishing for the hard $\phi^{4}$ potential. Finally, it has been found that for the $\phi^{4}$ potential with $\alpha=\pi / 2$, the (in)stability depends on the frequency even for infinitesimally small coupling, being additionally confirmed that a second-order perturbation theory is needed in order to predict the (in)stability at this particular value of the phase.

The above mentioned results open new perspectives for future work. For instance, we have started to study the properties of vortices in square lattices, which can be pictured as multibreathers with $\alpha=\pi / 2$. It can be interesting to apply the multibreathers stability theorem to two structures considered by Cretegny and Aubry: (i) breather "rivers" (percolating cluster) firstly and (ii) phonobreather with vacancy sites. Finally, we have also started to study the effect of long-range interactions in the stability of non-time reversible structures. 

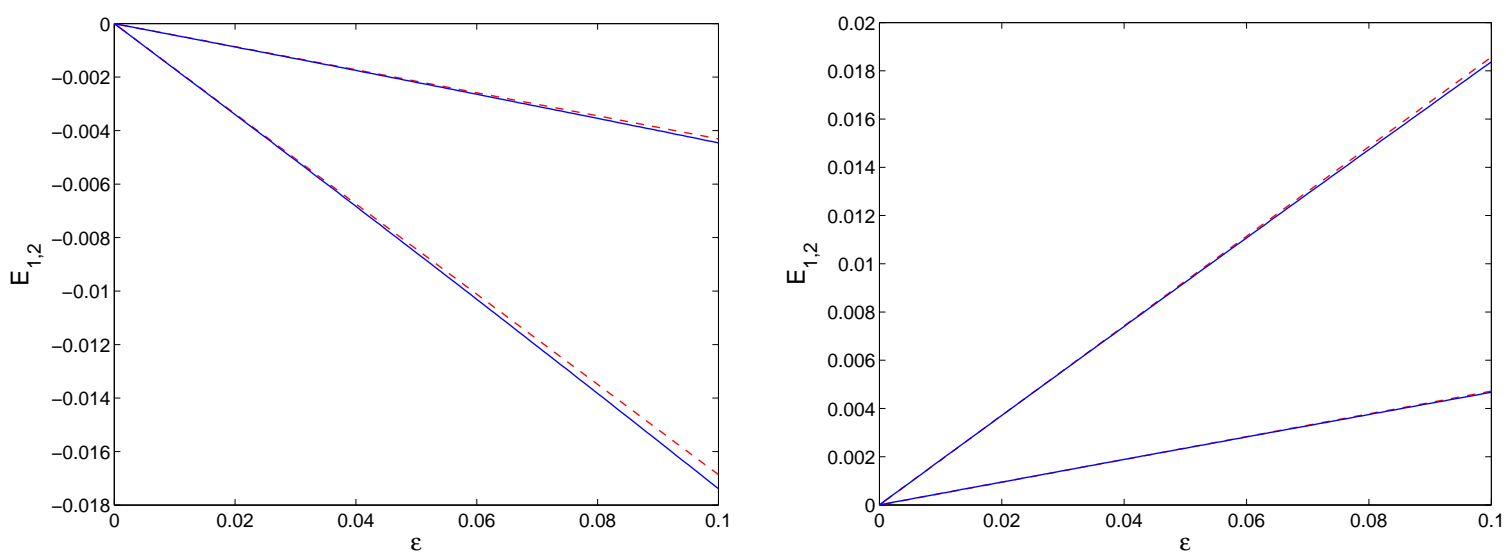

Figure 13. Dependence of the location of the maxima of the two bands closest to $E=0$ (represented by $E_{1}$ and $E_{2}$ ) with respect to $\varepsilon$ for phonobreathers in a $\phi^{4}$ with $\alpha=2 \pi / 3, N=21$ (left) and $\alpha=\pi / 4, N=24$ (right). In both cases, $\omega_{\mathrm{b}}=0.8$. Dashed lines correspond to the MST predictions and full lines to the numerical values. Dashed lines correspond to the MST predictions and full lines to the numerical values.
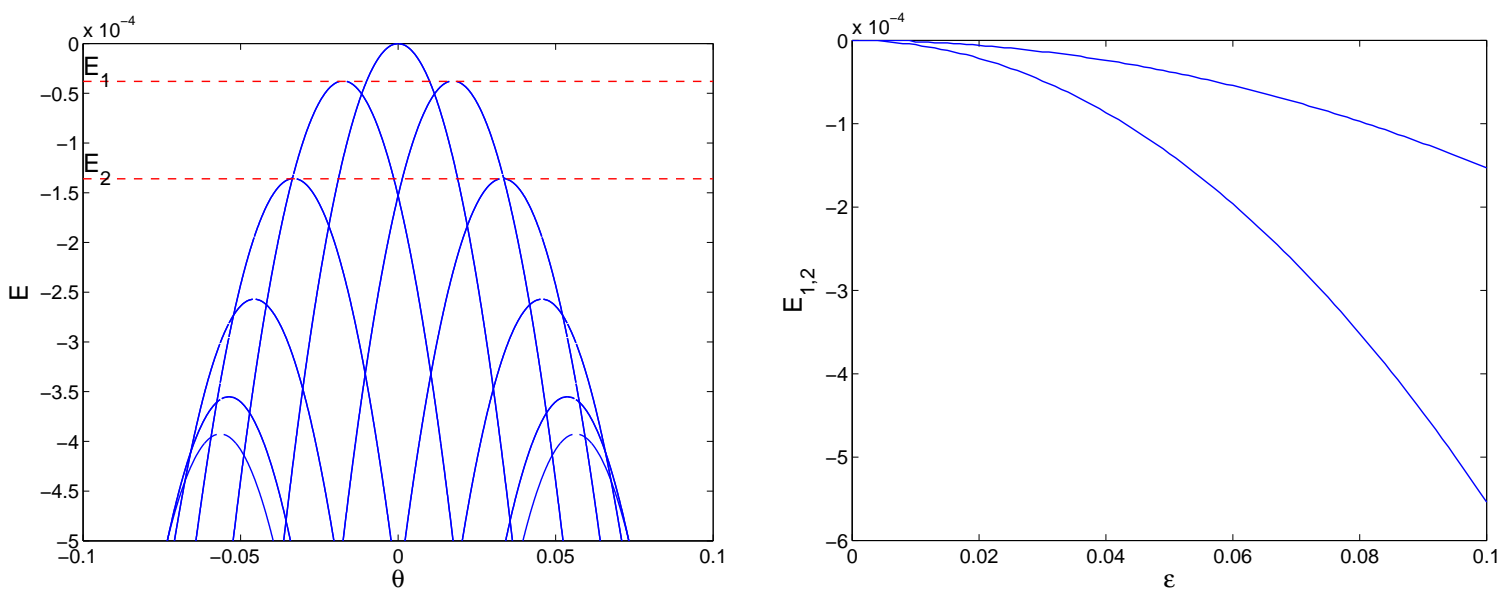

Figure 14. Phonobreather in a $\phi^{4}$ potential with $\omega_{\mathrm{b}}=2, \alpha=\pi / 2$ and $N=20$. (Left) Band diagram for $\varepsilon=0.05$. (Right) Dependence of the location of the maxima of the two bands closest to $E=0$ with respect to $\varepsilon$.

\section{Acknowledgments}

We acknowledge financial support from the MICINN project FIS2008-04848. 


\section{Appendix A. Calculation of the critical coupling}

In this appendix we calculate the critical value of the coupling parameter $\varepsilon$ for the on-site potentials considered in the paper, i.e. the Morse and the $\phi^{4}$ potentials, by making use of the rotating wave approximation (RWA) (see e.g. [44]). This method consists in considering all the Fourier coefficients zero but the first one, as this is usually considerably larger that the other coefficients. The RWA gives often surprisingly good results, taken in account its simplicity.

Let us consider equation Eq. (1):

$$
\ddot{u}_{n}+V^{\prime}\left(u_{n}\right)+\varepsilon\left(2 u_{n}-u_{n+1}-u_{n-1}\right)=0, \quad n=1, \ldots, N .
$$

We can write $u_{n}$ and $V^{\prime}\left(u_{n}\right)$ in terms of their fourier coefficients $z_{k, n}$ and $V_{n, k}^{\prime}$ as

$$
u_{n}(t)=\sum_{k=-k_{m}}^{k_{m}} z_{k, n} \exp \left(i k \omega_{\mathrm{b}} t\right) ; \quad V^{\prime}\left(u_{n}(t)\right)=\sum_{k=-k_{m}}^{k_{m}} V_{k, n}^{\prime} \exp \left(i k \omega_{\mathrm{b}} t\right)
$$

As $z_{k, n \pm 1}=z_{k, n} \exp ( \pm i \alpha)$, substituting in Eq. (A.1) we obtain

$$
-k^{2} \omega_{\mathrm{b}}^{2} z_{k, n}+V_{k, n}^{\prime}+4 \varepsilon z_{k, n} \sin ^{2}(\alpha / 2) .
$$

Let us calculate the coefficients $V_{k, n}^{\prime}$ for the generic $\phi^{4}$ potential $V\left(u_{n}\right)=\frac{1}{2} u_{n}^{2}+\frac{1}{4} s u_{n}^{4}$. Then $V^{\prime}\left(u_{n}\right)=u_{n}+s u_{n}^{3}$ or

$$
V^{\prime}\left(u_{n}\right)=\sum_{k=-k_{m}}^{k_{m}} z_{k, n} \exp \left(i k \omega_{\mathrm{b}} t\right)+s\left(\sum_{k=-k_{m}}^{k_{m}} z_{k, n} \exp \left(i k \omega_{\mathrm{b}} t\right)\right)^{3} .
$$

As $u_{n}(t)$ is real $z_{1, n}=z_{-1, n}^{*}$ and, therefore, within the RWA $u_{n}(t)=z_{1, n} \exp \left(i \omega_{\mathrm{b}} t\right)+z_{1, n}^{*} \exp \left(-i \omega_{\mathrm{b}} t\right)=$ $2\left|z_{1, n}\right| \cos \left(\omega_{\mathrm{b}} t+\phi_{1, n}\right)$ with $\phi_{1, n}=\arg \left(z_{1, n}\right)$. For a given $n=p$, we can always set $\phi_{1, p}=0$ by choosing the appropriate time origin; that is $z_{1, p} \in \mathbb{R}$ and $z_{1, p}=z_{-1, p}$. We only need to find the coefficients for the site $n=p$ as we can deduce any other one by using $z_{k, n+p}=z_{k, p} \exp (i n \alpha)$. Let us write $u(t) \equiv u_{p}(t), z_{k} \equiv z_{k, p}$ and so on, for simplicity. Then

$$
\begin{aligned}
& V^{\prime}(u(t))=\left(z_{1} \exp \left(i \omega_{\mathrm{b}} t\right)+z_{1} \exp \left(-i \omega_{\mathrm{b}} t\right)\right)+s\left(z_{1} \exp \left(i \omega_{\mathrm{b}} t\right)+z_{1} \exp \left(-i \omega_{\mathrm{b}} t\right)\right)^{3}= \\
& z_{1}\left(\exp \left(i \omega_{\mathrm{b}} t\right)+\exp \left(-i \omega_{\mathrm{b}} t\right)\right)+s z_{1}^{3}\left(\exp \left(i \omega_{\mathrm{b}} t\right)+\exp \left(-i \omega_{\mathrm{b}} t\right)\right)^{3}= \\
& z_{1}\left(\exp \left(i \omega_{\mathrm{b}} t\right)+\exp \left(-i \omega_{\mathrm{b}} t\right)\right)+s z_{1}^{3}\left(3\left[\exp \left(i \omega_{\mathrm{b}} t\right)+\exp \left(-i \omega_{\mathrm{b}} t\right)\right]+\left[\exp \left(3 i \omega_{\mathrm{b}} t\right)+\exp \left(-3 i \omega_{\mathrm{b}} t\right)\right]\right)= \\
& \left(z_{1}+3 s z_{1}^{2}\right)\left(\exp \left(i \omega_{\mathrm{b}} t\right)+\exp \left(-i \omega_{\mathrm{b}} t\right)\right)+s z_{1}^{3}\left(\exp \left(3 i \omega_{\mathrm{b}} t\right)+\exp \left(-3 i \omega_{\mathrm{b}} t\right)\right)
\end{aligned}
$$

Therefore, the coefficient of $\exp \left(i \omega_{\mathrm{b}} t\right)$ is $V_{1}^{\prime}=\left(z_{1}+3 s z_{1}^{2}\right)$. Substitution in Eq. (A.3) for $n=p$ (omitted) gives $-\omega_{\mathrm{b}}^{2} z_{1}+\left(z_{1}+3 s z_{1}^{3}\right)+4 \varepsilon z_{1} \sin ^{2}(\alpha / 2)=0$. This equation has two solutions $z_{1}=0$ and $z_{1}=\left(-\omega_{\mathrm{b}}^{2}+1+4 \varepsilon \sin ^{2}(\alpha / 2)\right)$, which becomes also zero for the critical value of the coupling parameter $\varepsilon$ given by:

$$
\varepsilon_{c}=\frac{\omega_{\mathrm{b}}^{2}-1}{4 \sin ^{2}(\alpha / 2)} . \quad\left(\phi^{4} \text { potential }\right)
$$

Therefore, $z_{1, p} \equiv z_{1}=0$ and so are $z_{1, n}$ and $u_{n}$ within the RWA. The flux $J_{0}$ also becomes zero, according to Eq. (10). This result does not depends on the value of $s$, which measures de degree of nonlinearity of the potential. As any hard symmetric potential can be approximated by the $\phi^{4}$ potential with some $s>0, \varepsilon_{c}$ is a good approximation for all of them. However, for a soft symmetric potential $s$ is negative, $\omega_{\mathrm{b}}<1$, and as $\varepsilon>0$ there is no value of the coupling that nullifies $z_{1}$ and the flux cannot be nullified. 
For the Morse potential, the RWA approximations is not so useful. From the numerical results we know that the critical coupling $\varepsilon$ corresponds to a divergence of the flux $J_{0}$, and therefore to a divergence of $u_{n}$ and its first fourier coefficient $z_{1, n}$. Then, $V^{\prime}\left(u_{n}\right)=2\left(\exp \left(-2 u_{n}\right)-\exp \left(-u_{n}\right)\right)$ tends to zero when $u_{n} \rightarrow \infty$ and so does $V_{1, n}^{\prime}$. By substitution in Eq. (A.3) for $k=1$, neglecting $V_{1, n}^{\prime}$ and simplifying $z_{1, n}$ we get $-\omega_{\mathrm{b}}^{2}+4 \varepsilon \sin ^{2}(\alpha / 2)=0$ and the critical coupling is

$$
\varepsilon_{c}=\frac{\omega_{\mathrm{b}}^{2}}{4 \sin ^{2}(\alpha / 2)} . \quad \text { (Morse potential) }
$$

We have not been able to deduce the reason why $u_{n}$ becomes infinity. 


\section{References}

[1] Aubry S 2006 Physica D 2161

[2] Flach S and Gorbach AV 2008 Phys. Rep. 4671

[3] MacKay RS and Aubry S 2004 Nonlinearity 71623

[4] Álvarez A, Archilla JFR, Cuevas J and Romero FR 2002 New J. Phys. 472

[5] Morgante AM, Johansson M, Kopidakis G and Aubry S 2002 Nonlinearity 16253

[6] Johansson M and Kivshar YuS 1999 Phys. Rev. Lett. 8285

[7] Aubry S 1997 Physica D 103201

[8] MacKay RS and Sepulchre JA 1998 Physica D 119148

[9] Archilla JFR, Cuevas J, Sánchez-Rey B and Álvarez A 2003 Physica D 180235

[10] Cuevas J, Archilla JFR and Romero FR 2005 Nonlinearity 18769

[11] Koukouloyannis V and Kevrekidis PG 2009 Nonlinearity 222269

[12] Ahn T, MacKay RS and Sepulchre JA 2001 Nonlinear Dyn. 25157

[13] MacKay RS and Sepulchre JA 2002 J. Phys. A: Math. Gen. 353985

[14] MacKay RS 2004 Slow manifolds (Energy Localisation and Transfer) ed T. Dauxois, A. Litvak-Hinenzon et al (Singapore: World Scientific) p 149

[15] Cuevas J, Koukouloyannis V, Kevrekidis PG and Archilla JFR 2010 Multibreather and vortex breather stability in Klein-Gordon lattices: Equivalence between two different approaches. Preprint. ArXiv:1006.0346.

[16] Flach S and Gorbach AV 2008 Lect. Notes Phys. 751289

[17] Cubero D, Cuevas J and Kevrekidis PG 2009 Phys. Rev. Lett. 102205505

[18] Marín JL, Falo F, Martínez PG and Floría LM 2001 Phys. Rev. E 63066603

[19] Martínez PG, Meister M, Floría LM and Falo F 2003 Chaos 13610

[20] Cuevas J, English LQ, Kevrekidis PG and Anderson M 2009 Phys. Rev. Lett. 102224101

[21] Sato M, Hubbard BE and Sievers AJ 2006 Rev. Mod. Phys. 78127

[22] English LQ, Basu Takhur R and Stearret R 2008 Phys. Rev. E 77066601

[23] English LQ, Palmero F, Sievers AJ, Kevrekidis PG and Barnak DH 2010 Phys. Rev. E 81046605

[24] Trías E, Mazo JJ and Orlando TP 2000 Phys. Rev. Lett. 84741

[25] Binder P, Abraimov D, Ustinov AV, Flach S and Zolotaryuk Y 2000 Phys. Rev. Lett. 84745

[26] Cretegny T and Aubry S 1997 Phys. Rev. B 55 R11929

[27] Cretegny T and Aubry S 1998 Physica D 113162

[28] Malomed BA and Kevrekidis PG 2001 Phys. Rev. E 64026601

[29] Cuevas J, Malomed BA and Kevrekidis PG Phys. Rev. E 76046608

[30] Law KJH, Kevrekidis PG, Koukouloyannis V, Kourakis I, Frantzeskakis DJ and Bishop AR 2008 Phys. Rev. E 78066610

[31] Cuevas J, James G, Kevrekidis PG and Law KJH 2009 Physica D 2381422

[32] Cuevas J, Hoq QE, Susanto H and Kevrekidis PG 2009 Physica D 2382216

[33] Koukouloyannis V and MacKay RS 2005 J. Phys. A: Math. Gen. 281021

[34] Flach S, Ivanchenko MV and Kanakov OI 2006 Phys. Rev. E 73036618

[35] Mishagin KG, Flach S, Kanakov OI and Ivanchenko MV 2008 New J. Phys. 10073034

[36] Marín JL and Aubry S 1996 Nonlinearity 91501

[37] Archilla JFR, MacKay RS and Marín JL 1999 Physica D 134406

[38] Marín JL 1999 Intrinsic Localized Modes in nonlinear lattices. PhD Thesis (University of Zaragoza)

[39] Cuevas J 2003 Localization and energy transfer in anharmonic inhomogeneus lattices PhD Thesis (University of Sevilla)

[40] Gradshteyn IS and Ryzhik IM 1965 Table of integrals, series, and products (New York: Academic Press)

[41] Kopidakis G and Aubry S 1999 Physica D 130155

[42] Khare A, Lakshminarayan A and Sukhatme U 2003 Local identities involving elliptic functions. Preprint ArXiV:mathph/0306028. This paper was published as Pramana 62 (2004) 1201, but the referred identity only appears in the preprint.

[43] Lawden DF 1989 Elliptic Functions And Applications (Berlin: Springer-Verlag)

[44] Page JB 1990 Phys. Rev. B 417835 